\title{
Braking Performance Analysis of Hydrodynamic Retarder Based on CFD
}

\author{
Fan Lidan ${ }^{1}$ \\ College of Mechanical Engineering \\ Jilin Teacher s' Institute of Engineering \& Technology \\ Changchun, China \\ Fanlidan111@163.com
}

\author{
Chen Youquan ${ }^{2}$ \\ Department of Mechanical Engineering \\ Changchun Institute of Engineering Technology \\ Changchun, China \\ Quan3168@sina.com
}

\begin{abstract}
To accurately predict the brake performance of hydrodynamic retarder, the computational fluid dynamics (CFD) is used to transient numerical simulation of liquid-gas two-phase flow in this paper. It obtained the distribution characteristic of pressure and velocity, and calculated the braking torque. On the basis, relation curves of braking torque and liquid filling rate are plotted. Comparison between simulation results and experimental data shows that simulation results are in good agreement with experimental data. It is indicated that predicting the performance of hydrodynamic retarder by the CFD simulation is accurate and practicable.
\end{abstract}

Keywords-hydrodynamic retarder ; braking performance; CFD; torque; braking time

\section{INTRODUCTION}

Hydrodynamic retarder is a kind of safely auxiliary braking device. It is widely used in heavy vehicles because advantages of high speed braking torque, low noise, compact structure etc. Hydrodynamic retarder are usually in a state of partial filling in participating in braking[1-3]. The internal flow field is gas-liquid two-phase flow. Prediction and calculation of braking performance is more complex than full liquid filling condition .

With the application of CFD technology in design of hydrodynamic components, domestic researchers have been research on braking process in the steady state of hydrodynamic retarder used CFD technology[4]. But the hydrodynamic retarder is the transient flow field in the actual operating conditions. When the vehicle is in emergency braking of state internal working chamber of hydrodynamic retarder is instantaneous oil filling process. The complex two-phase flow of internal rapidly changes with time as the gas-liquid volume[5-7]. The steady state calculation is unable to accurately describe in a state of dynamic balance of internal flow field of hydrodynamic retarder. Therefore, this paper focuses on the research of transient flow field of hydrodynamic retarder using CFD technology. Calculate the transient flow field Methods are used to numerical simulate dynamic characteristics of three dimensional, and the simulation results and experimental data were compared[8-10].

\section{CFD NUMERICAL SIMULATION}

\section{A. Governing Equations}

When luqid flow in internal hydrodynamic retarder is a continuous, three-dimensional, viscous and two-phase flow, the equation is:

$$
\frac{\partial \rho_{m}}{\partial t}+\nabla \cdot\left(\rho_{m} f_{m}\right)=0
$$

where $\tilde{v}_{m}$ is mass-average velocity,

$$
\rho_{m}=\frac{\sum_{k=1}^{n} \alpha_{k} \rho_{k} v_{k}}{\rho_{m}}, \mathrm{~m} / \mathrm{s} ;
$$

$\rho_{m}$ is mixture densities, $\rho_{m}=\sum_{k=1}^{n} \alpha_{k} \rho_{k}, \mathrm{~kg} / \mathrm{m}^{3}$;

$\alpha_{k}$ is the volume fraction of $k$ phase;

$\rho_{k}$ is the densities of $\boldsymbol{k}$ phase, $\mathrm{kg} / \mathrm{m}^{3}$ 。

Momentum equation can be acquired by summing all the momentum equation of respective phase. It can be expressed as :

$\frac{\partial}{\partial t}\left(\rho_{m} \rho_{m} f_{m}\right)+\nabla \cdot\left(\rho_{m} f_{m} \rho_{m}\right)=-\nabla p+\rho_{m} g+$

$\nabla \cdot\left[\mu_{m}\left(\nabla \dot{v}_{m}+\nabla \rho_{m}^{T}\right)\right]+\stackrel{\rho}{F}+\nabla \cdot\left(\sum_{k=1}^{n} \alpha_{k} \rho_{k} \rho_{d r, k} \rho_{d r, k}\right)$

where $n$ is phase number;

$F$ is volumetric force, $N$;

$\nabla$ is Hamilton operator;

$\mu_{m}$ is mixed viscosity,

$\mu_{m}=\sum_{k=1}^{n} \alpha_{k} \mu_{k}, \quad p a \cdot s ;$

$\hat{v}_{d r, k}$ is the drift velocity of phase $k, \quad \boldsymbol{v}_{d r, k}, \mathrm{~m} / \mathrm{s}$ 。

The equation of volume fraction of phase $p$

$\frac{\partial}{\partial t}\left(\alpha_{p} \rho_{p}\right)+\nabla \cdot\left(\alpha_{p} \rho_{p} \rho_{m}\right)=-\nabla \cdot\left(\alpha_{p} \rho_{p} \rho_{d r, p}\right)$

where $\alpha_{p}$ is the volume fraction of phase $p$;

$\rho_{p}$ is the densities of phase $p, k g / \mathrm{m}^{3}$; 
$\hat{v}_{m}$ is mass-average velocity,

$$
\rho_{m}=\frac{\sum_{k=1}^{n} \alpha_{k} \rho_{k} v_{k}}{\rho_{m}}, \mathrm{~m} / \mathrm{s} ;
$$

$\tilde{v}_{d r, p}$ is the drift velocity of phase $p, \quad \tilde{v}_{d r, p}=\tilde{v}_{p}-\tilde{v}_{m}$,

$\mathrm{m} / \mathrm{s}$ 。

\section{B. Model installment}

The model is set up according the lucite model of hydrodynamic retarder in experiment. The effective diameter of the circle of circulation is $\mathrm{D}=300 \mathrm{~mm}$. Stator and rotor blade number is $35 / 37$, blade ante version angle is 45 degrees. Fig .1(a) is three-dimensional model of hydrodynamic retarder. In order to get more accurately calculation, overall flow channel is adopted to analyze. The function flow channel of hydrodynamic retarder is formed by the space between the case bodies of both impeller and turbine and blades.(Fig .1(b)). Because the case bodies are not involving in counting, they can be ignored.

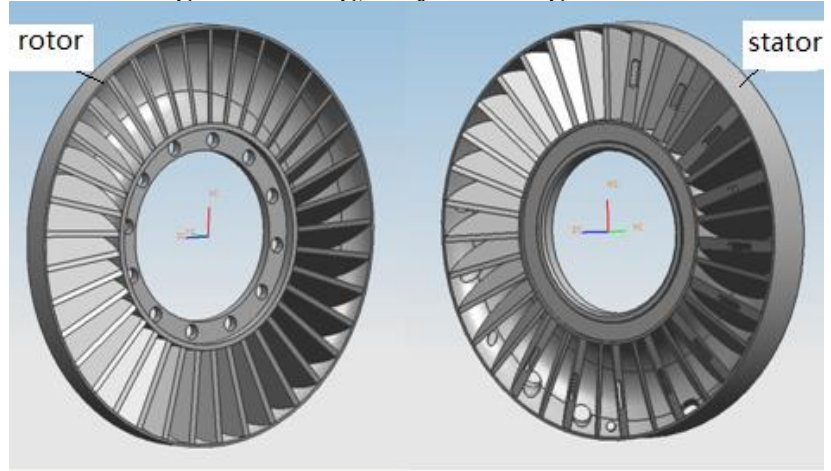

(a)

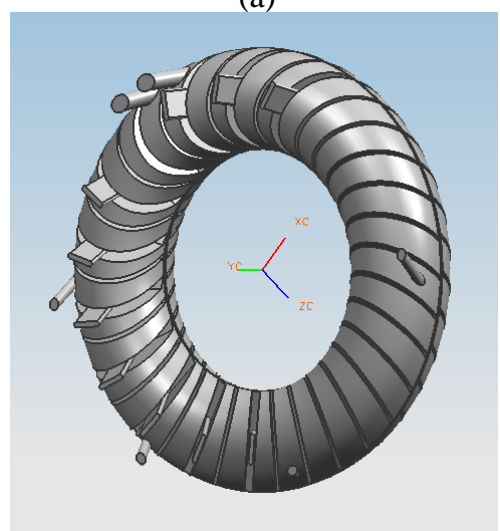

(b)

Figure 1. Three-dimensional modle of hydrodynamic retarder

Grid model is geometry expression of CFD model as well as the carrier of counting and analyzing. In order to get the grid model of flow pass, we first get the three-dimensional flow pass model from the impeller UG model and then lead that model in Gambit to divide grid, forming grid mode of flow pass for calculating. (Fig .2) The inside flow of hydrodynamic retarder is very unstable and there is strong interaction between the flow channel of pump and turbine. In order to make the unities calculation, sliding grid theory can be used to count the interface of the flow passes of rotor and stator. The paper regards the faying surface of rotor and stator as interface. Other surface is regarded as wall. After finished the settlement of the model, respectively do the calculation of the braking condition when the impute rotate speed is $1200 \mathrm{r} / \mathrm{min}$ while the liquid-filled rate are $25 \%$ and $75 \%$.

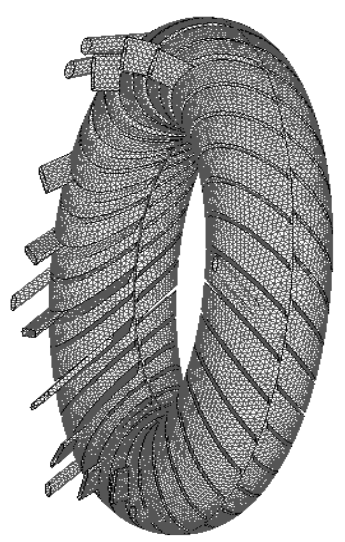

Figure 2. Grid model

\section{Analysis of flow field}

Fig .3 is the pressure scatter gram within the rotor flow channel when liquid filled rate is $25 \%$ and $75 \%$ under braking condition. I t can be seen that pressure values are layered increased gradually from inner to outside of blades. With the increase of liquid filling rate, blades of high pressure area expand and the highest pressure value increases. The maximum pressure value increases from $0.866 \mathrm{MPa}$ to $2.3 \mathrm{MPa}$. the lowest pressure value also increase from $-0.718 \mathrm{MPa}$ to $-1.93 \mathrm{MPa}$. So filling rate has a great influence on pressure distribution of the flow field .

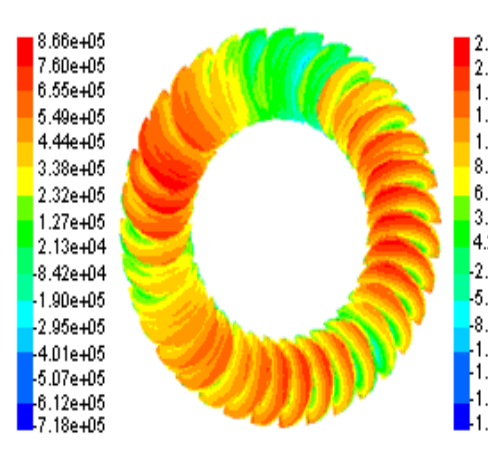

(a) $q_{c}=25 \%$
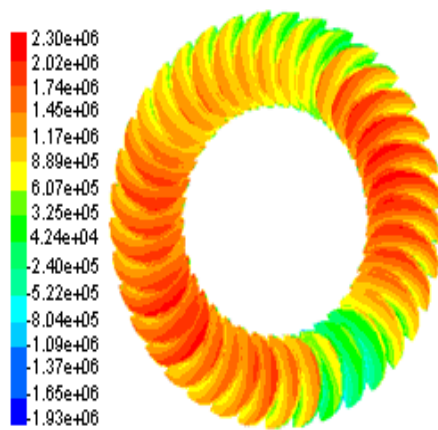

Figure 3. Contours of pressure of rotor

Fig .4 is contours of velocity vectors within the rotor flow channel when liquid filled rate is $25 \%$ and $75 \%$ under braking condition. As the figure shows that the highest value of velocity is $111 \mathrm{~m} / \mathrm{s}$ and the minimum velocity value is $0.697 \mathrm{~m} / \mathrm{s}$ under the filling rate scale of $25 \%$. While maximum was $113 \mathrm{~m} / \mathrm{s}$ and the minimum speed value is $0.558 \mathrm{~m} / \mathrm{s}$ under the filling rate of velocity scale $75 \%$. The average speed value in the $30.1 \mathrm{~m} / \mathrm{s} \sim 66.9 \mathrm{~m} / \mathrm{s}$.

Fig .5 is the pressure scatter gram within the stator flow channel when liquid filled rate is $25 \%$ and $75 \%$ under braking condition. The high pressure produces a high pressure area in the stator blade at the outer edge, the reason is the speed difference of the rotor and the stator is relatively large, so that the rotor speed is the outflow of the liquid by 
the great impact to the stator blades, thereby forming a high pressure area of the outer ring. Low pressure area is generated obvious near the circulatory circle at the center of the inner edge of the stator blades, the reason is the hydrodynamic retarder without inner ring, the stator and the rotor of the interface are especially liquid inflow and outflow condition in the center circle, it is easy to produce two secondary flow and vortex, so it is easy to form a low pressure area.

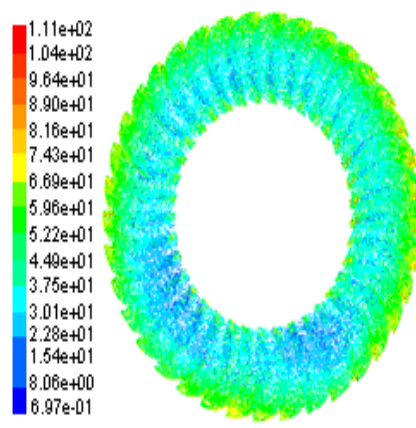

(a) $q_{c}=25 \%$
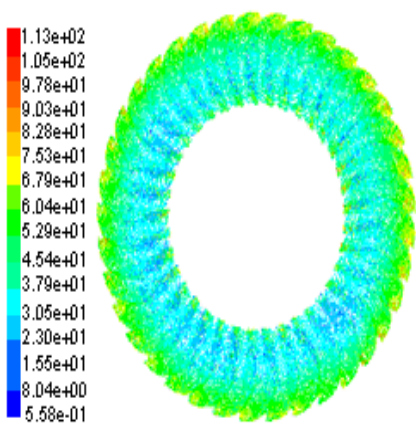

(b) $q_{c}=75 \%$
Figure 4. Contours of velocity vectors of rotor

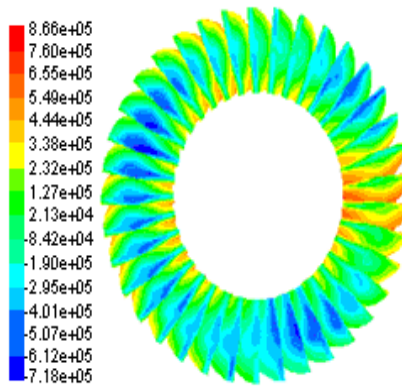

(a) $q_{c}=25 \%$
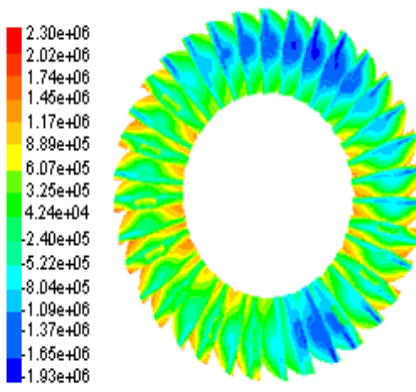

Figure 5. Contours of pressure of stator

Fig .6 is contours of velocity vectors within the stator flow channel when liquid filled rate is $25 \%$ and $75 \%$ under braking condition. Relative to the static pressure distribution of hydrodynamic retarder stator low speed region, corresponding to the low pressure area, near the blade inner edge of circular circle at the center of the velocity vector value in the $15.4 \mathrm{~m} / \mathrm{s}-30.1 \mathrm{~m} / \mathrm{s}$, due to the design of the hydrodynamic retarder oil inlet and outlet is arranged on the stator, so the low voltage distribution of stator vanes and low velocity distribution on the study and below is very important reference significance.

Fig .7 is a hydrodynamic retarder with different filling rate of the whole flow passage of the liquid volume distribution. Comparison of liquid filling rate of $\mathrm{q}=0.25$ and $q=0.75$, it can be found that the gas phase is mainly concentrated in the hydrodynamic retarder channel inner ring and liquid phase mainly concentrated in the channel of the outer ring. The vapor phase occupied most of the space work area under $25 \%$ liquid filled. The liquid phase occupied most of the space work flow when $75 \%$ the process of filling liquid.
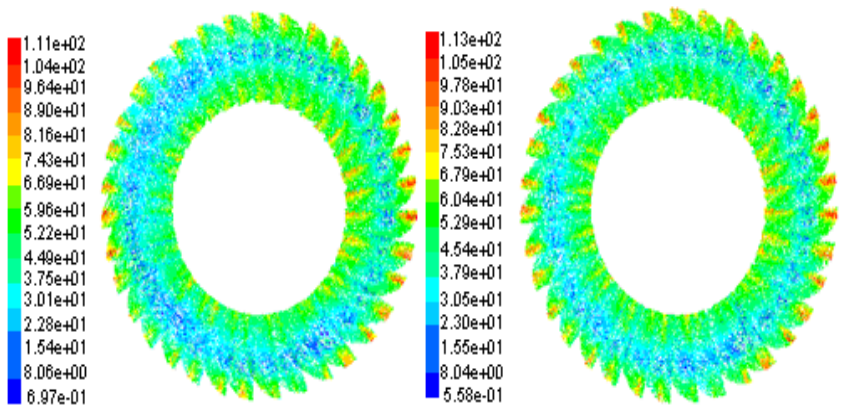

(a) $q_{c}=25 \%$

(b) $q_{c}=75 \%$

Figure 6. Contours of velocity vectors of stator
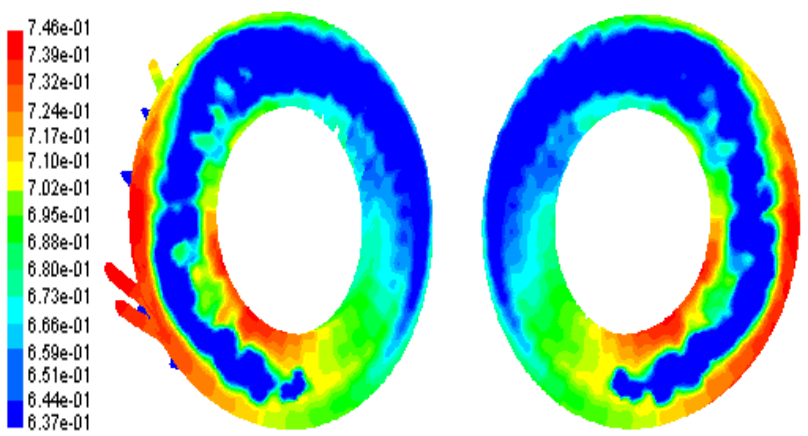

$q_{c}=25 \%$

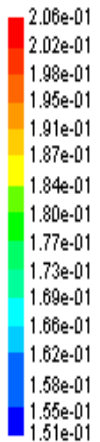

55 e.01

$$
q_{c}=75 \%
$$

Figure 7. Contours of volume fraction (liquid)

\section{PERFORMANCE ANALYSIS}

In the partially filled with liquid working conditions of the internal flow field of hydrodynamic retarder. Numerical simulation of three-dimensional flow field was designed to calculate the relation between braking torque and filling rate. Based on the simulation result, the curve of the relationship between the brake torque and filling rate as shown in Fig .8. As can be seen from the graph, the braking moment meet monotonically increasing law along with the increase of liquid filling rate under the rotor at different speed conditions. From the point of detail, brake torque variation gradient increases obviously during liquid filling rate in the interval of $80 \% \sim 100 \%$.

Select full filling condition, the hydrodynamic retarder prototype are the external characteristic experiment Because the restriction of experimental conditions the highest speed of the rotor is $500 \mathrm{r} / \mathrm{min}$. Comparisons 
between the simulation and the experiment curve as shown in Fig .9. The numerical results with the experimental values of the same change trend. Braking torque monotonically increased along with the rotor speed by two times curve. The maximal relative error of numerical results and the experimental results is $8.2 \%$

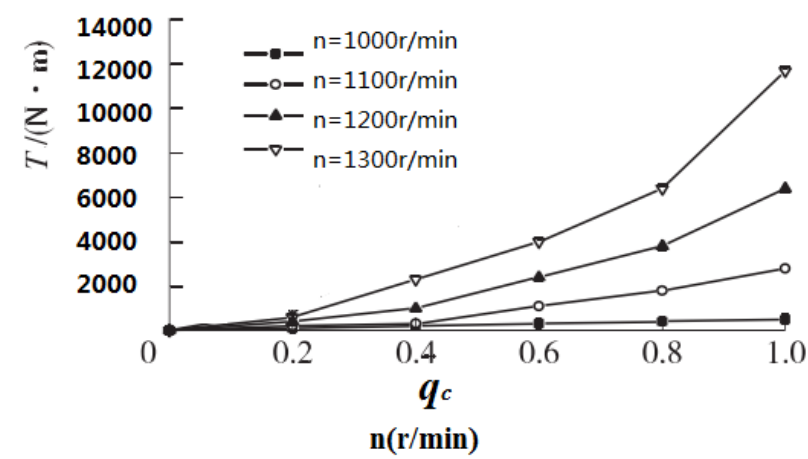

Figure 8. Curve of the relationship between brake torque and filling rate

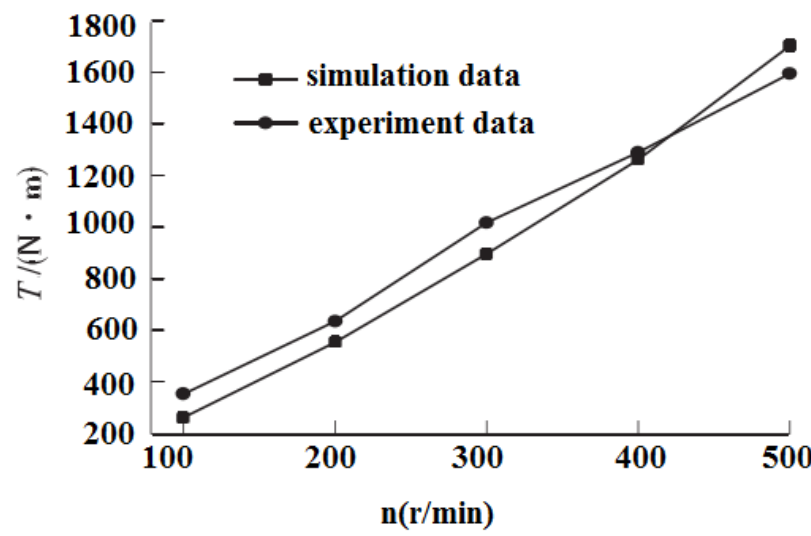

Figure 9. Comparisons curve between the simulation and experiment

\section{CONCLUSION}

The internal flow field of hydrodynamic retarder are studied by using the CFD technology in this paper. The change relationship is revealed between filling rate and braking torque. After experimental verification, the method used in this paper is reliable. It provide some theoretical basis for design and performance optimization of hydrodynamic retarder.

\section{REFERENCES}

[1] Wei Wei, Li Huiyuan, Zou Bo, et al. "Study on braking performance and analysis of two-phase flow in vehicular hydraulic retarder," in Transactions of Beijing Institute of Technology . vol.30(11), pp. 1281-1284, 2010.

[2] Wang Feng, Yan Qingdong, Qiao Jiangang. "The calculation method for braking performance of vehicular hydraulic retarder," in Hoisting and Coveying Machinery.vol 5,pp.24-27,2006.

[3] Yuan Zhe, Ma Menxing, Lu Xiuquan. "Dynamic braking performance prediction and analysis of hydrodynamic retarder," in Journal of jilin university. Vol.43,pp.160-164,2013.

[4] Yan Jun, He Ren. "Performance analysis for hydrodynamic retarter with different vanes," in Transactions of the chinese society for agricultural machinery. Vol.40,pp.206-209,2009.

[5] Li Xuesong. "Research on the parameter optimization method of automotive hydraulic retarder based on unsteady flow field analysis".Changchun: College of Automotive Engineering, Jilin University,2010.

[6] Yan Qing-dong, Wei Wei. "Numeric Simulation of Single Passage Ternary Turbulence Model in Hydraulic Torque Converter," in Journal of Beijing Institute of Technology (S1004-0579), vol.12(2),pp. 172-175.2003.

[7] Song Bin, Lv Jiangang, Liu Yun, et al.“The Simulation and Analysis on Engine and Hydraulic Retarder Continua,". Proc. The Ninth International Conference on Electronic Measurement \& Instruments Press, Mar.2009,pp:928-931.

[8] Ma Wenxing, Liu Chunbao, Wu Kaifu. "Numerical Simulation and Analysis on the Control of Flow Separation in Hydrodynamic Coupling", Proc. Conference Proceedings of 5th International Symposium on Fluid Power Transmission and Control, 2007.

[9] Zhu Jingchang, Wei Chenguan, Zheng Muqiao, "Vehicle hydraulic transmission: upper and lower volume" National Defense Industry Press, 1982.

[10] Yao Shouwen, Wang Xiaolong, "Optimization on blade grid system of hydraulic reducer and braking dynamics simulation," in Journal of Machine Design,vol.12,pp.21-23,2007. 